\title{
RECUPERAR LA MEMORIA VISUALIZANDO NUESTRO PATRIMONIO ARQUEOLÓGICO ${ }^{(*)}$
}

\author{
RECOVERING MEMORY BY VISUALIZING OUR ARCHAEOLOGICAL HERITAGE \\ PALOMA CARCEDO DE MUFARECH ${ }^{(*)}$ Y MARIO ADVÍNCULA ZEBALLOS $^{(* \star *)}$
}

Fecha de recepción: 04 de junio de 2015

Fecha de aprobación: 14 de junio de 2015

\section{RESUMEN}

El actual territorio de Lima Metropolitana tiene un admirable patrimonio precolombino, obras que son testimonios de miles de años de constante transformación de un territorio árido en un verdadero oasis en la franja desértica de la costa peruana. Sin embargo, los monumentos arqueológicos de Lima han sido históricamente excluidos de la memoria de la ciudad; no forman parte de la "limeñidad" en el imaginario de su población. De tal manera, las huacas no son consideradas como parte de la formación ciudadana, ni contempladas dentro de las políticas de desarrollo territorial de Lima Metropolitana. Por el contrario, son consideradas como espacios abandonados y peligrosos. El presente artículo reflexiona sobre esta problemática y las acciones que se tomaron en torno a la gestión del patrimonio arqueológico desde la Gerencia de Cultura de la Municipalidad Metropolitana de Lima entre los años 2011 y 2014, periodo en que se desarrolló el programa Lima Milenaria, Ciudad de Culturas, que conllevó, además de la recuperación de edificios prehispánicos, la implementación de un proyecto educativo y actividades que involucraron a la comunidad con sus huacas.

\section{PALABRAS CLAVE}

Patrimonio arqueológico, memoria, territorio

\section{ABSTRACT}

The current territory of Lima has an impressive pre-Columbian heritage, works that bear witness to thousands of years of constant transformation of a barren land into a true oasis in the desert belt of the Peruvian coast. However, archaeological monuments in Lima have been historically excluded from the memory of the city, and have not been part of the "limeñidad" in the minds of its people. So it is that the huacas are not considered a part of civic education, nor taken into account in the territorial development policies of Lima, but, considered dangerous and abandoned spaces. The present article reflects on this problem and the actions taken for the management of archaeological heritage by the Management of Culture of the Metropolitan Municipality of Lima from 2011 to 2014, a period in which the Millennial Lima, City of Culture program was developed, which led, not only to the recovery of prehispanic buildings, but, also, to the implementation of an educational project and activities which involved the community with its huacas.

\section{KEYWORDS}

Archaeological heritage, memory, territory

(*) El presente artículo desarrolla la problemática en torno a la gestión del patrimonio arqueológico desde la Gerencia de Cultura de la Municipalidad Metropolitana de Lima entre los años 2011 y 2014, periodo en el que se desarrolló el programa Lima Milenaria, Ciudad de Culturas, el cual conllevó, además de la recuperación de edificios prehispánicos, la implementación de un proyecto educativo y actividades que involucraron a la comunidad con sus huacas.

$\left.{ }^{(* *}\right)$ Historiadora, museóloga, curadora de exposiciones nacionales e internacionales, especialista en metalurgia andina Precolombina y gestora cultural. Doctora en Historia del Arte por la Universidad Complutense de Madrid (España). Magíster en Museum Studies por la Universidad de Nueva York (EE.UU.). Ha sido docente de la Pontificia Universidad Católica del Perú, directora general de Patrimonio Cultural del Ministerio de Cultura y subgerente de Patrimonio Cultural, Artes Visuales, Museos y Bibliotecas de la Municipalidad Metropolitana de Lima. Actualmente ejerce la docencia en la Universidad de Lima y es Directora General de Cooperación Internacional de la Biblioteca Nacional.

(***) Arqueólogo graduado en la Universidad Nacional Mayor de San Marcos. Diplomado en Museología en la misma universidad, con especialización en Políticas Culturales y Gestión Cultural en la Universidad Metropolitana de Iztapalapa (México). Su trabajo se ha dedicado a los temas de museología, gestión cultural y territorio. Ha sido jefe de la división de Patrimonio Arqueológico de la Subgerencia de Patrimonio Cultural, Artes Visuales, Museo y Bibliotecas de la Municipalidad Metropolitana de Lima del año 2012 hasta 2014, donde ejerció como coordinador de las actividades que giraron en torno al programa Lima Milenaria, Ciudad de Culturas. Actualmente es director del Proyecto de tramo Vilcashuamán-Pisco del Qhapaq Ñan-Sede nacional. 
Los conceptos de patrimonio cultural y memoria histórica están a menudo presentes en cerrados debates culturales y discursos políticos, así como en alguna que otra conferencia especializada. Pero, realmente, el ciudadano de a pie, el alumno o profesor universitario o, incluso, las personas que trabajan en hacer textos escolares ¿entienden la importancia de estos conceptos para nuestra formación como ciudadanos? O, más aún, ¿para ejercer ciudadanía? De ser así, ¿alguien pensaría en tirar la basura en una huaca, desaparecerla del mapa porque hay que hacer un túnel o construir las casas aledañas de espalda a estas? En nuestro imaginario histórico visualizamos Lima como una ciudad colonial; no nos imaginamos que hasta 1950 tenía más de 600 monumentos arqueológicos, conocidos como huacas, algunos con más de 4000 años de antigüedad -por lo que se ha merecido el gran nombre de "milenaria"-, y que estas complejas y ricas culturas transformaron el territorio: dejaron no solo extraordinarios vestigios arquitectónicos, sino un rico bagaje cultural. Los valles de Lima han sido escenario del desarrollo humano desde el año 7000 a.C., cuando las lomas y el mar cumplieron un importante rol para la subsistencia de las primeras poblaciones. Sin embargo, es a partir del año 2200 a.C., con el desarrollo de grandes centros ceremoniales, como El Paraíso en el valle de Chillón, que Lima adquiere un rol cada vez más protagónico. En los milenios siguientes estos complejos sobrevivieron a continuos embates, hasta que, en el siglo XX, ante la desatada y poco planificada fiebre constructora de la ciudad, empezaron a desaparecer, hecho que fue investigado y reportado en varias publicaciones (por ejemplo, Agurto, 1984; Ravines, 1985).

En el territorio que ocupa actualmente Lima Metropolitana, florecieron diversas y complejas culturas formadas por gentes procedentes de diversos puntos de los Andes Centrales, que se asentaron al margen de los tres valles que la forman: Rímac, Lurín y Chillón. Desde épocas pretéritas este espacio territorial fue un crisol de culturas donde se mezclaron y fusionaron cultos, costumbres, tecnologías y cosmovisiones que en el avatar histórico enriquecieron esta tierra y, a su vez, irradió su cultura a otros puntos del país, creando identidades propias. Por lo tanto, se trata de un territorio que fue un lugar de encuentro y fusión de gentes de la sierra y de la costa, del norte y del sur, del este y del oeste, que fueron quienes dibujaron la Lima en que vivimos.

Esta riqueza cultural quedó plasmada, entre otras cosas, en los monumentos arquitectónicos que como mudos testigos han llegado hasta nosotros para recordar lo que fuimos. Los estudios arqueológicos realizados en los últimos años dan cuenta de una Lima pluricultural y pluriétnica, formada por las diferentes sociedades que compartían este territorio como un gran lugar de encuentro y co-existencia. Hoy en día, es común oír que vivimos en un mundo globalizado, donde las grandes urbes han tomado la forma de comunidades transnacionales y/o cuentan con una gran población migrante; Lima Metropolitana no es la excepción. Los más de 9 millones de habitantes que ocupan su territorio son migrantes procedentes de los más diversos puntos del país. Pero esto, como se ha mencionado, no es algo nuevo, sino, más bien, una práctica ancestral, embrión de lo que ahora somos. Si en el pasado poblaciones de orígenes diferentes pudieron compartir un territorio respetándose mutuamente, ¿por qué no ahora? Si diferentes grupos étnicos -como los huatqas, malanqas, amanqaes, latis, sulqos- compartieron el valle de Lima, y han dejado no solo sus nombre en la toponimia local, sino su manera de entender el mundo, ¿por qué no reflexionamos sobre cómo tratar el patrimonio que hemos heredado, para ser mejores ciudadanos? Patrimonio cultural y ciudadanía son términos que encierran muchos conceptos. Lima ocupa un territorio redibujado desde épocas ancestrales, el cual hoy continua transformándose mediante ricas y diversas expresiones culturales, cuya complejidad contribuyó en las últimas décadas a conformar el territorio metropolitano que hoy conocemos, dividido en las cuatro Limas -como los cuatro suyos del Tahuantinsuyo-: Lima Este, Lima Norte, Lima Centro y Lima Sur.

1. En el video de Lima Cultura (video de la Gerencia de Cultura de la Municipalidad Metropolitana de Lima) (Febrero 2012) Proyecto de Investigación, Conservación y Puesta en Valor de la Huaca Palomino. Recuperado de https://www.youtube.com/watch?v=7LZEbnBNkj0 
Nuestra tarea, desde las instancias públicas y privadas, debe ser reconocer esta riqueza y diversidad cultural, ancestral y actual, e interactuar con estas distintas comunidades, no solo respetando su identidad y patrimonio cultural, sino apropiándonos de él, dándolo a conocer mediante el diálogo y aprendizaje mutuo -ya sea mediante buenos programas de educación o el registro de su patrimonio-, para así lograr que nos sintamos orgullosos de la herencia y del presente que nos identificará a todos como ciudad y país ante el mundo. Necesitamos mirar a Lima Metropolitana como una ciudad milenaria, pero también la queremos planificada y cuidada. Queremos que las generaciones futuras puedan contemplar y admirar sus imponentes vestigios arqueológicos mediante un desarrollo urbano consciente y coherente, que incluya los monumentos arqueológicos que hoy en día, como mudos testigos, se resisten a ser olvidados. Debemos aprender a mirar Lima como una ciudad de culturas diversas, en donde sus ciudadanos sepan conocerse y reconocerse, no solo luchando por sus derechos económicos, civiles y políticos, sino también sociales y culturales. Debemos formar ciudadanos que entiendan y respeten las diferentes culturas con las que cohabitan, pero también aquellas que cohabitaron este territorio; solo entonces aprenderemos a respetarnos nosotros.

Es muy poco común que los propios limeños, o quienes visitan Lima, la visualicen como una ciudad milenaria con cientos de pirámides "embutidas" en ella. ¿Cuántas ciudades del mundo se conoce que albergan más de 365 monumentos arquitectónicos precolombinos o precoloniales? Si tal es nuestra riqueza precolombina arquitectónica, ¿por qué tanta desidia en cuidarla a lo largo de tantas décadas? La respuesta es compleja. Por una parte, no se valora lo que no se conoce, y por años nuestro patrimonio arquitectónico precolombino ha estado "oculto" bajo un manto de arena, basura y falta de conocimiento, incluso para los propios pobladores de las zonas aledañas; por otra parte, las autoridades edilicias se han negado a entender lo que es y lo que implica un monumento arqueológico y, por ende, en la mayoría de los casos, no lo hacían formar parte de su catastro.

Un dato curioso es que en 2012, mientras revisábamos los planos del Parque de Las Leyendas -el cual encierra más de 50 monumentos arquitectónicos precolombinos-, vimos con sorpresa que en el plano topográfico del área que manejaba la Municipalidad de San Miguel no estaban considerados los monumentos arquitectónicos precolombinos que se encuentran dentro, sino que esos terrenos figuraban como áreas baldías o verdes. Gracias a la intervención de la dirección de arqueología del Parque de Las Leyendas -la cual depende de la Municipalidad Metropolitana de Lima- y en coordinación con el Ministerio de Cultura, a finales de 2012 se empezó una campaña de registro de todos los monumentos arquitectónicos precolombinos, de tal manera que hoy en día están ya 53 registrados con el levantamiento georeferencial y visado por el Ministerio de Cultura. Esto, que a simple vista no parece de gran importancia, sí la tiene, pues sino estuvieran registrados literalmente se podría haber pasado un Caterpillar ${ }^{2}$ por ellos para construir, por ejemplo, un parque de diversiones estilo Hollywood con casinos u hoteles, barbaridad de la que nuestras autoridades políticas son capaces. La poca "rentabilidad" de un monumento hace que no se quiera reconocer como patrimonio a muchos de ellos, para así tener el camino abierto para construcciones más rentables. Para entender la "rentabilidad" de poner en valor un monumento arquitectónico, basta el ejemplo de la huaca Palomino en el Cercado de Lima. En 2012 la Municipalidad Metropolitana de Lima, a través de la Gerencia de Cultura, la puso en valor; lo que antes era un basural con casas circundantes que le mostraban su rostro más precario, es ahora un lugar que los vecinos y vecinas están orgullosos de mostrar, que consideran parte de su historia y que fortalece su pertenencia a una tierra. En el sentido más "económico" del asunto, sus predios han duplicado o triplicado su precio, aunque, por desgracia, muchas veces son sus cocinas y no sus salas las que miran al monumento iluminado y cuidado.

2. Tal como ocurrió en 2013 con el monumento arquitectónico precolombino El Paraíso. 
Cuando los especialistas solicitan fondos y presentan presupuestos bien estructurados al MEF (Ministerio de Economía y Finanzas), o a cualquier organismo público o privado, se enfrentan a un calvario en donde los más sólidos argumentos se desvanecen al intentar hacer comprender al funcionario que el patrimonio no se puede ver como un "gasto sin retorno".

Pero, ¿de que réditos estamos hablando?, ¿culturales?, ¿económicos? ¿Por qué no los dos? Desde los grandes pensadores griegos como Aristóteles (384-322 a.C.) hasta el filósofo norteamericano Arthur Danto (1924), el tema de cómo la cultura o el arte afecta o no a los ciudadanos ha sido tema de debate. Aristóteles comentaba que el $\operatorname{arte}^{3}$ es un válvula de escape de las emociones. No hace falta que sea útil sino que dé felicidad (Hauskeller, 2008, p. 20). Siglos más tarde, el poeta, dramaturgo y filósofo alemán Friedrich Schiller (1759-1805) comentaba que "El arte no puede mejorar de manera inmediata a las personas pero puede devolverles la posibilidad para lo bueno convirtiendo lo necesario y eterno en objeto de sus impulsos" (Hauskeller, 2008, p. 41). Para muchos pensadores, por ejemplo Schiller, el arte o la cultura no puede asediar en vano sus máximas morales pero puede influir en sus ocios (Hauskeler, 2008, p. 41). Es decir, la cultura nos puede ayudar en nuestra manera de ser y pensar.

Ejemplos muy claros son Medellín (Colombia) y Madrid (España), donde la cultura ha cambiado barrios enteros. Teniendo esto presente es que la gestión de la alcaldesa Susana Villarán creó la Gerencia de Cultura y, dentro de esta, subgerencias que se dedicaban de lleno a transmitir y brindar cultura a la ciudad de Lima. Por primera vez, la cultura entraba al escenario político y ciudadano. Es en este escenario que se crea, en conjunto con el Ministerio de Cultura, un programa de puesta en valor de monumentos arquitectónicos precolombinos, donde el principal esfuerzo se centra en que los vecinos y vecinas de estos se apropien de los mismos, porque, a la postre, serán ellos quienes de manera efectiva se proclamarán sus guardianes. El camino es lento pero, una vez iniciado, esperamos no haya retroceso.

Los programas de puesta en valor de complejos arquitectónicos deben de ir acompañados de programas educativos bien estructurados, como los que se hicieron desde la Gerencia de Cultura (Vamos a las Huacas y El Patrimonio no está Pintado), en los que participaban colegios de toda la ciudad. Asimismo, en el programa Talleres de Arte y Arqueología, participaban los colegios aledaños a los sitios arqueológicos, cuyos docentes eran capacitados y recibían publicaciones de manera gratuita sobre cómo replicar dichos talleres a sus alumnos y demás profesores. Estas publicaciones eran compartidas también en la web de la Municipalidad Metropolitana de Lima para su libre descarga.

Todo lo realizado, que en un principio parece una lucha quijotesca, se ve de repente fortalecido por una brisa de aliento cuando empiezan a surgir un número de iniciativas privadas para la conservación del patrimonio monumental arqueológico que, poco a poco, van extendiéndose por diferentes distritos. Entre ellos está el Colectivo Colli en Comas, la Asociación Cultural Salvemos las Huacas, y el Instituto de Cultura, Historia y Medio Ambiente (ICHMA). Las iniciativas municipales y privadas se han dado cuenta de que salvando nuestro patrimonio arqueológico nos salvamos a nosotros mismos de perder nuestra historia y la de su memoria para nuestros hijos. Además, nuestro patrimonio nos ayuda a comprender de dónde venimos y hacia dónde vamos, a conocernos y entendernos más como un país que encierra una rica historia de la cual sentirnos orgullosos y, en definitiva, a ser mejores ciudadanos. ¿Cómo cuantificamos económicamente ser mejores personas y sentirnos un pueblo orgulloso de nuestra historia? Quizás algún pensador del siglo XXI pueda ayudar a brindar un razonamiento lógico a esta pregunta.

\footnotetext{
3. En esas épocas arte era sinónimo de cultura.
} 


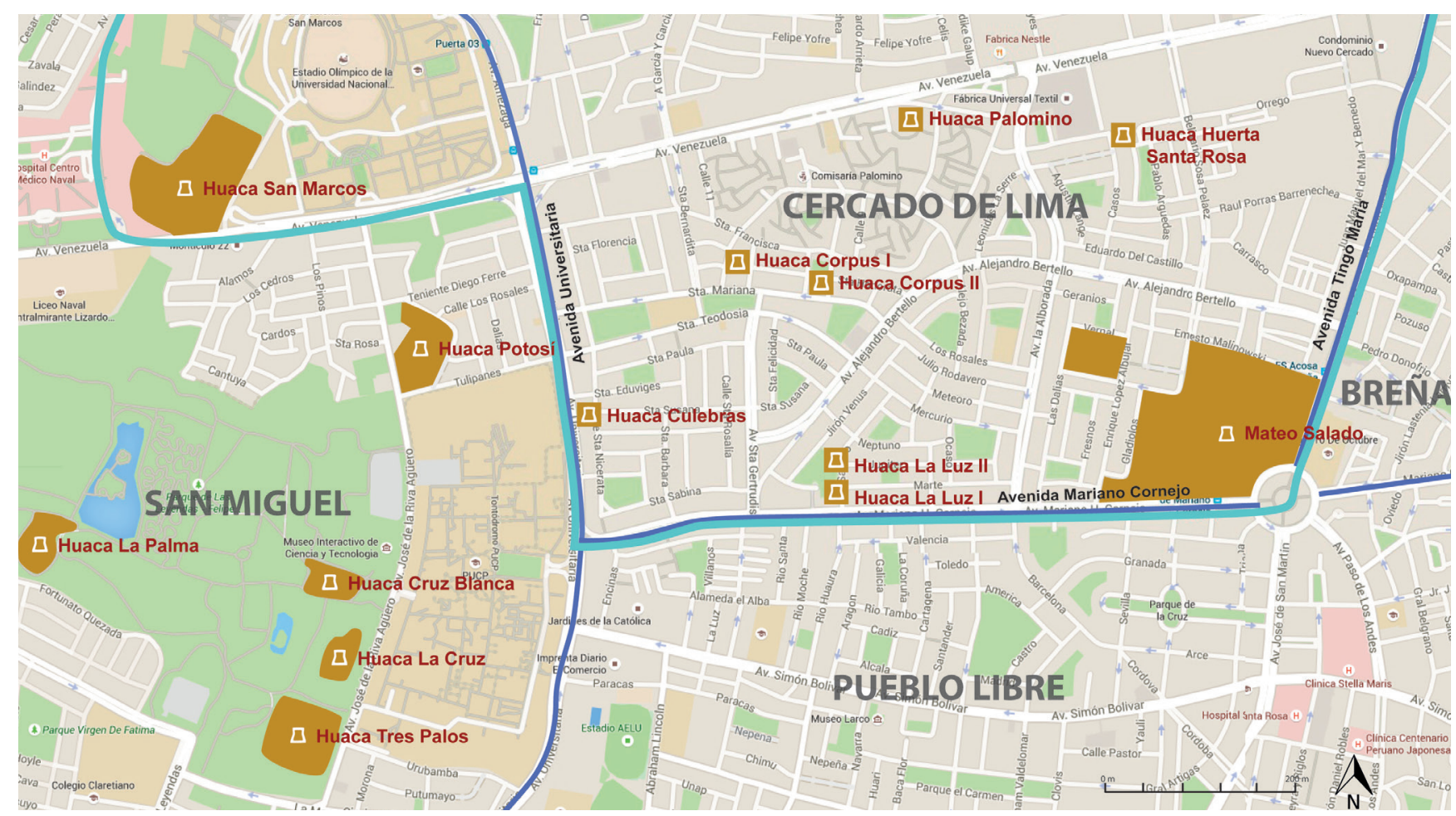

Los proyectos de recuperación del patrimonio arqueológico realizados por la Municipalidad Metropolitana de Lima entre los años 2012 y 2014 se ejecutaron dentro del programa Lima Milenaria, Ciudad de Culturas, el cual sigue los lineamientos de la "Declaratoria de Alcaldía 001/2012", que considera de interés prioritario la protección, conservación, investigación y difusión del patrimonio cultural de la ciudad, y supone la promoción de su conservación e integración a los espacios urbanos de la ciudad de Lima.

A partir de este concepto, la Gerencia de Cultura de la Municipalidad Metropolitana de Lima inició un programa que centró su acción en los sitios arqueológicos de la jurisdicción del Cercado de Lima, distrito en el cual se encuentran nueve zonas arqueológicas declaradas como Patrimonio Cultural de la Nación (Figura 1).

\section{Antecedentes de los Proyectos Arqueológicos}

Durante la década del 60, cuando el crecimiento urbano de la ciudad alcanzó las zonas de Pando y Palomino (al este del Centro Histórico de Lima), se realizaron varios proyectos arqueológicos dirigidos por el Seminario de Arqueología del Instituto Riva Aguero-PUCP, los cuales evidenciaron el carácter arqueológico de los montículos que quedaron delimitados dentro de la construcción de la nuevas urbanizaciones. Las huacas intervenidas fueron La Luz 1, La Luz 2, Corpus I, Corpus II, Culebras, Palomino (Cárdenas, 1970, 1973; Corbacho, 1970, 1971; Ramos de Cox, 1970). En la Figura 2 se puede apreciar la excavación arqueológica de la huaca Palomino.

Por su parte, desde hace más de una década, el Ministerio de Cultura (antes Instituto Nacional de Cultura) ejecuta proyectos de puesta en valor de sitios arqueológicos. Un ejemplo de ello es el proyecto de puesta en valor del sitio arqueológico Mateo Salado (Figueroa 2007, 2008; Espinoza 2009, 2013). Este proyecto no solo ha dado importantes resultados desde el punto de vista de la investigación, sino, también, en la habilitación de recorridos turísticos, lo que coadyuvó a desarrollar una importante agenda de actividades culturales, cuyo centro y escenario es el espacio arqueológico.
Figura 1. Sitios Arqueológicos del Cercado de Lima.

Fuente. Imagen redibujada del google maps, 2015, 
devenir Vol. 2, N³, ENERO - JUNIO 2015, PP. 99-112 - ESTUdIOS I ISSN 2312-7570

UNIVERSIDAD NACIONAL DE INGENIERÍA, LIMA

Figura 2. Construcción de la Residencial Palomino.

Fuente. Foto DIRAF - FAP, 1965
Por otra parte, otra institución que ha apoyado en la tarea de poner en valor nuestro patrimonio arqueológico es Plan COPESCO Nacional. Gracias a su participación se han ejecutado proyectos de conservación y de infraestructura -cerco y habilitación de senderos para el recorrido- en varios sitios arqueológicos del Cercado, como son las huacas La Luz I y La Luz II.

El Cercado, en realidad, es un área privilegiada de Lima que cuenta con importantes monumentos arqueológicos prehispánicos. Estos se pueden circunscribir en dos áreas importantes: el complejo Maranga, que abarca desde el actual Parque de la Leyendas hasta la Universidad Nacional Mayor de San Marcos, y el gran sitio Mateo Salado (entre los distritos de Pueblo Libre, Breña y Cercado de Lima). Entre las dos áreas se encuentran más de 365 huacas "embutidas" en la trama urbana, caso excepcional en el mundo si se considera que Lima es una capital que cuenta con más de 10 millones de habitantes y tiene un gran problema de espacios urbanos. Entre los sitios monumentales habría que señalar la huaca Aramburú o San Marcos, que es uno de los edificios de mayor monumentalidad del Complejo arqueológico Maranga, el cual se encuentra dentro de los predios de la Universidad Nacional Mayor de San Marcos. Hay que tener en cuenta que desde hace años y hasta la actualidad es intervenido como escuela de campo de los estudiantes de arqueología de la Universidad de San Marcos.

La mayoría de las huacas son de escala menor si se comparan con el carácter colosal de algunos de los monumentos mayores que se encuentran tanto en Mateo Salado como en el Complejo Arqueológico de Maranga. En tal sentido, considerando esta desigualdad y teniendo en cuenta que solamente se disponía de dos años para que terminara la gestión Municipal, se diseñó un plan de recuperación de huacas de Lima Metropolitana que se concentraba en la recuperación de sitios arqueológicos factibles de realizar a mediano plazo. En primer lugar tomaba en cuenta las del Cercado de Lima y, una vez concluido esto, seguía con otros distritos de Lima. Para ello era imprescindible tener como aliado al Ministerio de Cultura y, más específicamente, a la Dirección General de Arqueología. Ambas instituciones acordaron diseñar un plan de trabajo conjunto al que

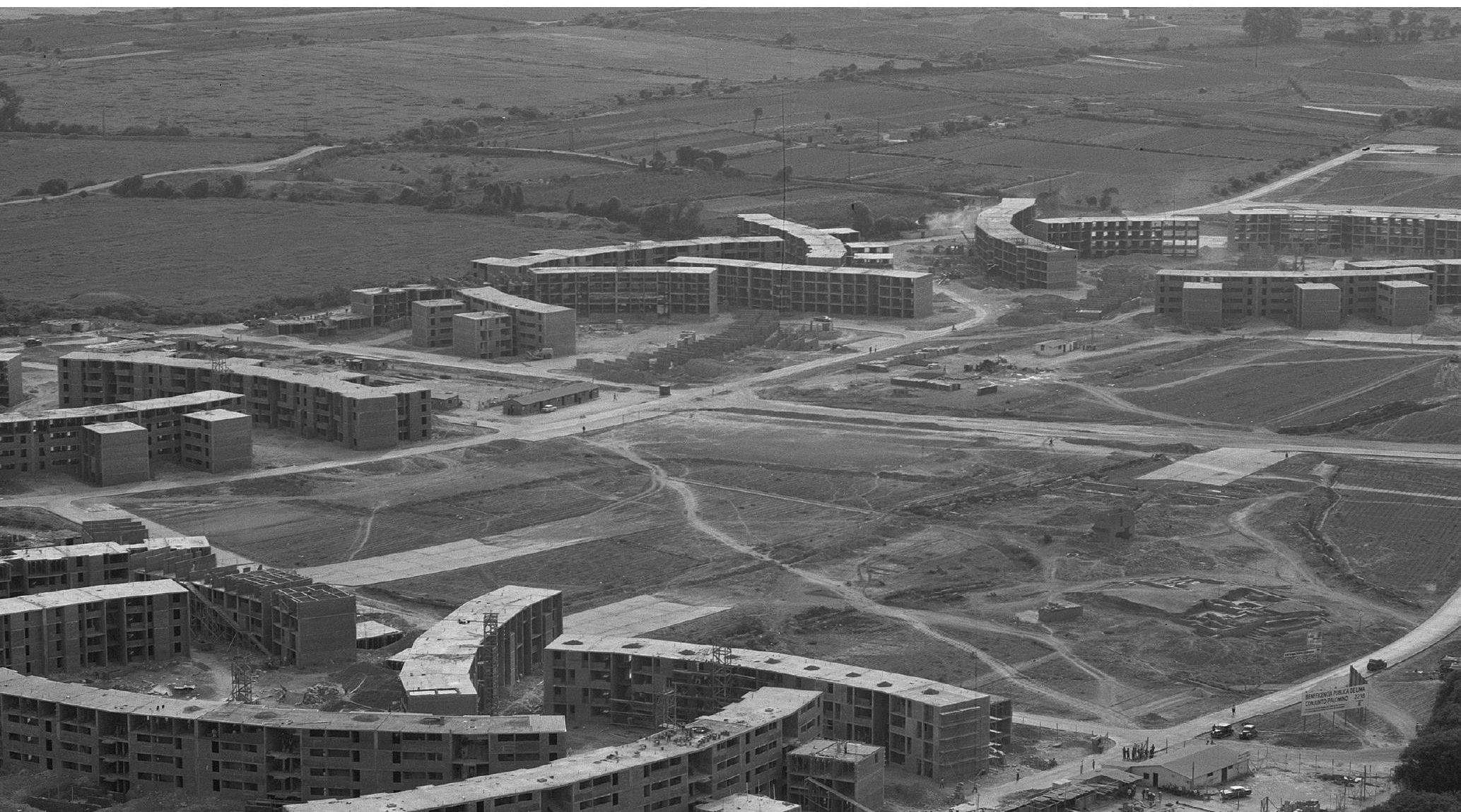


se llamó Mi Huaca. Herencia que nos une, el cual tuvo como colofón la firma de un convenio de cooperación institucional entre el Ministerio de Cultura y la Municipalidad Metropolitana de Lima, es decir, entre la Ministra de Cultura, Diana Álvarez-Calderón, y la alcaldesa, Susana Villarán, firmado en enero de 2014. La formalización de esta importante alianza tuvo como marco el espléndido sitio de la huaca Mateo Salado; destacaban entre sus principales objetivos el recuperar, proteger, investigar, poner en valor, difundir y promover el uso social y responsable de los monumentos arqueológicos en la capital, así como la realización de otras actividades que contribuyeran a la regeneración urbana de los espacios públicos y la revitalización del vecindario. Ambas entidades se comprometieron a convocar a las comunas distritales para sacar adelante el plan, así como organizar y participar de las labores de vigilancia de los sitios arqueológicos de Lima Metropolitana. Es decir, sentar las bases de un verdadero programa de gestión del patrimonio arqueológico de Lima Metropolitana, con una clara proyección a futuro.

Este plan trajo consigo también la realización de diversos foros Lima Milenaria en 2012 y en 2014, cuyo objetivo fue reflexionar sobre la gestión del patrimonio arqueológico y la participación ciudadana en su preservación.

\section{Proyectos de Puesta en Valor y Objetivos de los Proyectos Arqueológicos}

Los proyectos de puesta en valor de los sitios arqueológicos de Lima Metropolitana contemplaron cuatro objetivos fundamentales que se resumen no solo en el aspecto de conservación, sino, también, en el de crear espacios públicos vivos:

- Investigación y conservación de la zona arqueológica: La intervención realizada buscó establecer los elementos que nos permitieran determinar la secuencia de ocupaciones, así como las funciones que pudo tener el sitio. Asimismo, se realizó el diagnóstico del estado de conservación de la arquitectura expuesta del sitio y las labores necesarias para garantizar la conservación del monumento.

- Implementación de senderos y miradores: En los sitios arqueológicos intervenidos se habilitó un sistema de rampas y senderos que permiten el acceso a miradores para así poder apreciar el monumento, y las áreas excavadas y conservadas.

- Establecer un guión interpretativo de recorrido de la zona arqueológica: Una vez concluida la investigación, se elabora, en conjunto con el área educativa de la Gerencia de Cultura, un guión interpretativo del monumento, es decir, se preparan temáticas pedagógicas que permitan a los visitantes la interpretación del monumento.

- Propiciar la regeneración urbana del espacio arqueológico como espacio público: Una vez recuperada el área arqueológica, se inició una serie de actividades que promueve el uso público del espacio arqueológico. Estas actividades debían incluir visitas guiadas, talleres de arte y arqueología, funciones de títeres, cuenta cuentos, malabares, cine, teatro y música. Los principales beneficiarios eran los vecinos de la huaca, quienes no solo veían la recuperación del monumento histórico, sino su uso como espacio público para actividades culturales.

\section{Proyecto de Investigación, Conservación y Puesta en Valor de la Huaca Palomino 4}

La huaca Palomino se encuentra ubicada a la altura de la cuadra 27 de la Av. Venezuela, en la Residencial Palomino, Cercado de Lima. Su ocupación corresponde a la época ychsma (1000-1400 d.C.). Su actual emplazamiento resulta bastante estratégico e interesante, ya que el diseño de la Residencial Palomino, hecho por Luis Miró Quesada G., Santiago Agurto Calvo, Fernando Correa Miller y Fernando Sánchez Griñán durante los

4. El Lic. Piero Guarisco Canseco, arqueólogo de la Gerencia de Cultura de la MML, dirigió la ejecución de todos los proyectos de investigación y puesta en valor de la huacas Palomino, Corpus I, Corpus II y Culebras. 
devenir Vol. 2, N³3, ENERO- JUNIO 2015, PP. 99-112 - EsTUDIOS I ISSN 2312-7570

UNIVERSIDAD NACIONAL DE INGENIERÍ, LIMA

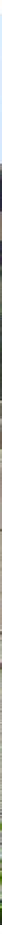

Figura 3. Foto huaca Palomino luego de su puesta en valor.

Fuente. Foto Mario Advíncula, 2013. años 1965 y $1966^{5}$ permitió conservar un área perimétrica libre, así como la implementación de un parque próximo. Además, los bloques de vivienda giran y contemplan la zona arqueológica. Es decir, el diseño de la urbanización dio un rol protagónico a la huaca, la cual además se encuentra frente a una importante avenida de la ciudad, que comunica al Centro de Lima con el Callao.

La huaca Palomino es un montículo de planta rectangular, de aproximadamente $30 \mathrm{~m}$ de largo por $20 \mathrm{~m}$ de ancho, con una altura máxima de $2.50 \mathrm{~m}$, construida mediante una sucesión de estructuras de tapial. Por su ubicación está asociada al Complejo arqueológico de Maranga, cuyo núcleo se encuentra en el Parque de las Leyendas (Guarisco, 2012).

Sin embargo, el abandono de la huaca hizo que este espacio sea utilizado como un basural, y considerado como un espacio peligroso y una preocupación permanente para los vecinos, quienes lo percibían como un foco negativo para la Residencial Palomino.

En ese sentido, era importante iniciar las acciones de recuperación de las huacas de El Cercado de Lima en Palomino, cuyo entorno urbano las ponía en alta prioridad.

En el mes de febrero del año 2012, la MML empezó con la ejecución del Proyecto de Investigación, Conservación y Puesta en Valor de la Huaca Palomino, con el que se logró la intervención en la cima plataforma, que puso en evidencia una sucesión de patios y banquetas a los cuales se accede luego de pasadizos y recintos que previamente restringían el acceso (Guarisco, 2012).

El proyecto contempló también el manejo del entorno del monumento. Por cuestiones de seguridad era necesario establecer un barrera en el perímetro del área arqueológica; sin embargo, colocar un cerco de concreto o rejas generaría un distanciamiento entre los vecinos y el sitio arqueológico, por lo que se procedió a implementar un 
cerco vivo del arbusto mioporo (especie recomendada por el Ministerio de Cultura). Asimismo, se implementó un sendero de visita, y se reactivó un sistema de iluminación desde los postes de luz.

\section{Proyecto de Investigación, Conservación y Puesta en Valor de la Huaca Corpus I}

La huaca Corpus I se localiza en la III Etapa de la urbanización Pando (Cercado de Lima), entre las calles Santa Francisca Romana, Santa Justina y Santa Gertrudis, y el lado sur del sitio colinda con la parte posterior de dos inmuebles. El perímetro del área arqueológica tiene un pequeño muro que servía de base para una malla perimétrica que se encuentra destruida.

La huaca contiene una plataforma construida con tapiales de unos $2 \mathrm{~m}$ de altura, dos muros perimétricos hacia los lados sur y norte, y un muro de barro de edificación moderna que delimita el lado oeste de la plataforma. Este incluso se proyecta zigzagueante hacia el Norte de la misma y conforma una suerte de terraplén de unos $25 \mathrm{~m}$ de largo (Guarisco, 2014). El sitio se encuentra en el interior de la urbanización, a unos 300 m de la Av. Venezuela.

El estado de abandono de la huaca convirtió el espacio en un basural y lugar para covachas de personas indigentes, por lo que el concepto negativo (sucio y peligroso) era la referencia de los vecinos hacia el sitio arqueológico.

El año 2013 la Municipalidad Metropolitana de Lima ejecutó el Proyecto de Investigación y Puesta en Valor de la Huaca Corpus I. A través de este, se logró la recuperación del sitio, así como identificar dos ocupacionales correspondientes al periodo Intermedio Tardío, y, en particular, a la cultura Ychsma (Guarisco, 2014).

En la arquitectura destaca el primer momento constructivo, que está constituido por dos grandes patios rectangulares orientados por un acceso en la parte norte. Este espacio rellenado por cantos rodados y arena se constituyó como la plataforma principal, que correspondería al segundo momento constructivo, por una amplia plataforma de dimensiones aproximadas de $16.9 \mathrm{~m}$ de largo por $22.10 \mathrm{~m}$ de ancho. Sobre esta plataforma se han identificado ocho recintos y diez plataformas menores (Guarisco, 2014).

\section{Proyecto de Investigación, Conservación y Puesta en Valor de las Huacas Culebras y Corpus II}

La huaca Culebras se ubica en el Cercado de Lima, a la altura de la cuadra 16 de la Av. Universitaria (frente de la Pontificia Universidad Católica del Perú), en la cuadra 5 de la calle Santa Nicerata, urbanización Santa Emma, tercera etapa. El área corresponde a una zona debidamente urbanizada.

La huaca Corpus II se encuentra localizada en Cercado de Lima, entre las calles Santa Coleta y Perpetuo Socorro, en la urbanización Pando, tercera etapa. Se encuentra $160 \mathrm{~m}$ al oeste de la huaca Corpus I.

Ambas huacas colindan con inmuebles al norte y sur, y al este y oeste con vías de tránsito (Figura 1). Precisamente, los lados colindantes con las calles presentan muros y la puerta de acceso. En el caso de la huaca Culebras solo se puede acceder desde la calle Santa Nicerata, y para el caso de la huaca Corpus II se accede a través de la calle Santa Coleta.

El área circundante de los montículos de cada uno de estos sitios se mantiene como un jardín, un espacio verde cuidado por los vecinos. Sin embargo, el estado de conservación de los sitios se veía afectado por la humedad y la vegetación que estaba muy próxima a los muros del montículo.

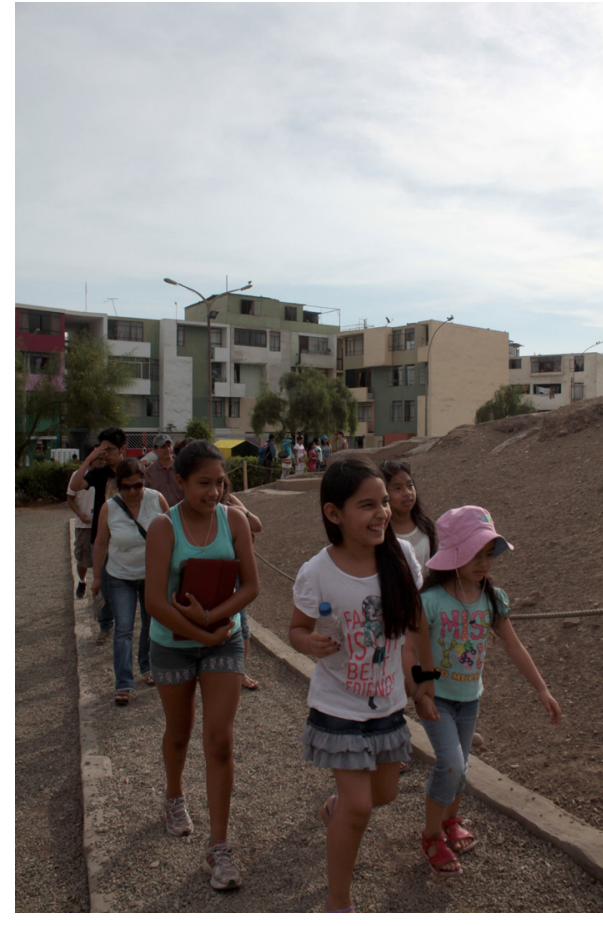

Figura 4. Foto de visitas guiadas en la huaca Palomino.

Fuente. Foto Gerencia de Cultura de la MML, 2013. 
En vista de ello, la intervención en ambos monumentos tuvo como prioridad la conservación del monumento arqueológico, así como mantener el perímetro con el área verde.

Si bien la huaca Culebras fue intervenida en la década del 60 por el Instituto Riva Agüero, se requería de una nueva intervención urgente para realizar la conservación del montículo, el cual se encontraba deteriorado por la exposición al medio ambiente. Para ello fue necesario excavar todo la superficie del montículo, a fin de analizar su arquitectura, y aplicar los tratamientos de conservación y consolidación que garanticen la exposición de la arquitectura.

La huaca Corpus II también fue excavada por el Instituto Riva Agüero, en la década del 60 ; entonces se registraron varios entierros y elementos culturales del periodo formativo. Al momento de iniciarse los nuevos trabajos, se podía observar un montículo de cantos rodados y tierra sin muros ni otros elementos arquitectónicos en superficie, lo que hizo necesario intervenir a fin de definir los elementos arquitectónicos del montículo y poder elaborar un guión interpretativo del sitio.

Como resultado de las excavaciones se ha definido, en el caso de la huaca Culebras, una sucesión de estructuras elaboradas con tapial que corresponden a la ocupación yschma y, posiblemente, inca (Guarisco, 2015).

En el caso de la huaca Corpus II, las excavaciones pudieron definir una sucesión de muros de contención de elaborados en base a cantos rodados cuya antigüedad correspondería a la cultura lima.

\section{Recuperación del Espacio Público Precolombino y su Contemporaneidad en el Uso}

Pocas veces identificamos el espacio público con las áreas que ocupan las llamadas huacas o monumentos arquitectónicos precolombinos en Lima Metropolitana. Ante todo porque estos han sido "ocupados" por construcciones, en un principio clandestinas, sin respeto por los límites arqueológicos, a vista y paciencia de las autoridades edilicias. Pero en realidad, nuestros "urbanistas" precolombinos no se limitaron a diseñar una huaca como si fuera una construcción aislada, sino que pensaron en ella inmersa en, e íntimamente unida a, un área más compleja, que comprendía un espacio abierto en el cual se realizaban diferentes actos, ya sean rituales (funerarios, religiosos, etc.) o de importancia para la comunidad (conmemoración de alguna batalla, etc.). De tal manera, este llegaba a ser el punto de encuentro más importante de la comunidad, lugar de afirmaciones políticas, religiosas y comunales. Siempre habrá excepciones, pero no es lo común. Por lo tanto, el pueblo, en determinadas ocasiones y, es de suponer, con el debido orden, hacía uso de este espacio y lo cuidaba como suyo porque, en el fondo, era un lugar de afirmación de su identidad.

Con esto en mente, es que durante el año 2012 la Municipalidad Metropolitana de Lima decidió llevar a cabo un plan piloto en la huaca Palomino (ubicada en la Av. Venezuela, Cercado de Lima), con el objetivo de no solo recuperar el monumento arqueológico, sino también el espacio abierto enfrente de este: una pequeña plaza que le devolviera su carácter de uso comunal. De esta forma, la ciudad de Lima no solo recuperaba un monumento arquitectónico precolombino, sino que la comunidad de El Cercado recibía un espacio en el cual se podían realizar actividades que propiciara la autoridad edilicia. Empero, la Gerencia de Cultura tenía claro que, al ser algo nuevo, habría que diseñar programas y proyectos que no solo enseñaran a la comunidad como debería utilizar este espacio, sino, también, las bondades de su utilización como lugar en el que se desarrollen programas educativos relacionados con nuestro patrimonio cultural. Por ello, ese mismo año, se convocó a especialistas en educación, y se empezó a diseñar programas que involucraran tanto el cuidado y conocimiento del patrimonio arqueológico como del inmaterial. Había que empezar con los colegios cercanos a estos $y$, fundamentalmente, con sus profesores. 


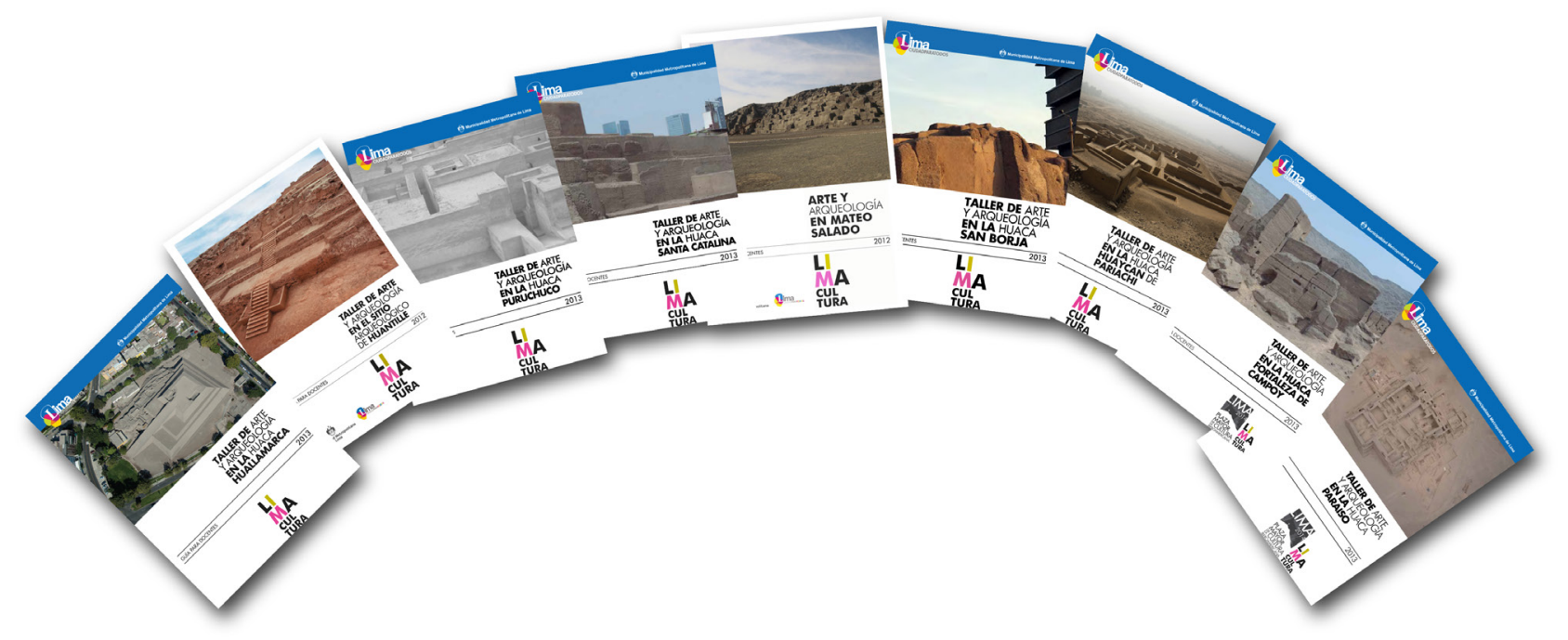

Bajo la dirección de Sandra Rospigliosi, y más tarde de Eibby Rosillo, se diseñó un Área de Programas Educativos que dependía de la Gerencia de Cultura y de la Subgerencia de Patrimonio Cultural, Artes Visuales, Museos y Bibliotecas. Su finalidad fue proponer, coordinar y diseñar un plan anual de actividades y programas educativos, y elaborar y ejecutar propuestas pedagógicas, las cuales involucraban también a la Pinacoteca Municipal Ignacio Merino, y el Museo Metropolitano o MET. El objetivo era, entre otras cosas, realizar actividades con los colegios cercanos a zonas arqueológicas -algunas en las cuales ya se había iniciado los trabajos para su puesta en valor-, y coordinar con los directores y docentes para explicar al alumnado de forma didáctica y amena la importancia de estos sitios arqueológicos: quiénes fueron sus "constructores", cuál era su función y la importancia del patrimonio inmaterial inherente de estos antiguos pueblos.

Para ello, se diseñaron los Talleres de Arte y Arqueología, Talleres de Arte y Patrimonio y los programas Vamos a las Huacas y Vamos a los Museos. La idea de estos talleres era que sirvieran de plataforma que invitara a los vecinos y comunidad en general a descubrir la importancia de la huaca. En un principio, se enseñaba de manera didáctica la historia arqueológica del sitio, de tal manera que, profesores primero y alumnos después, pudieran entender aquel monumento que, a pesar de formar parte de su "paisaje" urbano, no pasaba a formar parte de su imaginario cultural y menos a identificar al vecino con el pueblo que lo construyó. Ante el éxito del primer taller, realizado en la huaca Huantille en 2012, se realizaron más talleres, exactamente 10 hasta 2014. A partir de ese año, los talleres se llamaron Talleres de Arte y Patrimonio, ya que en estos talleres no solo se explicaba sobre la arqueología, sino, también, sobre el patrimonio inmaterial involucrado a la cultura que construyó dichos sitios. Así se realizaron talleres en Cantagallo, Barrios Altos y Huachipa en 2014. Cada taller intervenía durante un mes un determinado sitio arqueológico e involucraba a su población. Las actividades del taller incluían visitas gratuitas a sitios arqueológicos para alumnos y docentes de colegios públicos de Lima Metropolitana, además del transporte, talleres previos y material pedagógico para profesores y alumnos. La idea final era que los talleres sirvieran como guía a los docentes. Para ello, se colgó en la página web de la Municipalidad las 12 publicaciones que se realizaron, de tal manera que pudieran ser descargadas y fueran accesibles para todos los profesores de todas las unidades educativas, así como para el público en general.
Figura 5. Guía para docentes de los Talleres de Arte y Arqueología.

Fuente. Municipalidad Metropolitana de Lima, 2015. Lima Cultura. Programas educativos. Recuperado de http:// www.limacultura.pe/taxonomy/ term/654) 
Para los años 2013 y 2014, se decidió que los espacios arqueológicos recuperados podían ser utilizados de manera consciente por la comunidad que había visto poco a poco su recuperación. Para ello se empezaron a promocionar actividades y jornadas artísticas, en especial en la huaca Palomino, que incluyeron desde cuenta cuentos, títeres y malabares hasta espectáculos de música y cine, como Celebra Palomino, Cine en tu Huaca o Huacas, Burbujas y Rock and Roll, todos con gran éxito participativo (Figura 5).

\section{Hacia el Futuro con el Pasado}

La recuperación de las huacas Palomino, Corpus I, Corpus II y Culebras ha beneficiado a más de 30 mil vecinos del Cercado de Lima (entre la urbanización Palomino, Pando III etapa y Santa Emma III etapa). Hoy los vecinos de estas urbanizaciones cuentan no solo con la recuperación de terrenos considerados baldíos como las huacas Palomino y Corpus I, sino que los talleres educativos en los colegios del entorno han permitido que los estudiantes y docentes puedan acercarse al monumento para realizar jornadas pedagógicas. Asimismo, las asociaciones de vecinos han encontrado un referente en un espacio cultural público como parte de su urbanización.

Poder medir económicamente cuanto han aumentado el valor de los predios alrededor en estas urbanizaciones luego de las recuperación de las huacas es una tarea aún pendiente, pero necesaria. También se requiere medir el impacto social de la recuperación del sitio arqueológico, cuál es el grado de percepción y valoración del vecindario sobre sus monumentos arqueológicos recuperados, cómo los aspectos de seguridad y convivencia han cambiado y, finalmente, cómo ha cambiado su percepción de sí mismos.

Pensamos que esta parte del Cercado de Lima representa una gran oportunidad para convertirla en un recorrido turístico interesante $y$, al mismo tiempo, continuar con los programas educativos y las actividades culturales en las huacas. Además, existen las condiciones para integrar un circuito de espacios arqueológicos por parte del Ministerio de Cultura, como las huacas La Luz I, La Luz II y Mateo Salado, y tener una lectura histórica del territorio.

Los resultados obtenidos de esta experiencia piloto refuerzan la postura de la sostenibilidad de los proyectos de desarrollo en Lima Metropolitana, los cuales deben contemplar la riqueza monumental del pasado precolombino, no solo como parte de propuestas turísticas, sino como componente esencial para la formación de ciudadanía, así como para el ordenamiento y desarrollo territorial.

Entre las herramientas de gestión necesarias para lograr dichos fines está el saneamiento físico legal de los terrenos arqueológicos, aspecto imprescindible para su preservación y para la ejecución de Proyectos de Inversión Pública. Es este un punto álgido en el proceso de lograr la formalización de los predios y de nuestro patrimonio.

De igual manera, se requiere del trabajo conjunto y planificado (mediante convenios) entre las instituciones públicas, como el Ministerio de Cultura y las municipalidades, a fin de darle continuidad a los proyectos de puesta en valor. Acertadamente, estos proyectos ya no solo se enfocan en la conservación y restauración del espacio precolombino, sino en la posibilidad de que los sitios recuperados se integren como espacios públicos al entorno urbano mediante actos culturales e intervenciones artísticas.

La visualización del patrimonio arqueológico requiere de un trabajo sostenido en la gestión cultural de parte de las instituciones del gobierno municipal en coordinación con el Ministerio de Cultura. Los avances en ese sentido han sido importantes; sin embargo, se requiere de un compromiso claro de las autoridades e instituciones involucradas para no dejar de lado este avance en la gestión del patrimonio arqueológico, así como la determinación de todos los ciudadanos por defender su memoria histórica. 
Por otra parte, es imprescindible que los planes municipales de desarrollo y planificación urbanística involucren el patrimonio arqueológico con consenso de todas las bancadas y sean discutidos por especialistas, personal técnico y representantes de la comunidad. Es importante que, una vez aprobado, se mantenga su ejecución sin importar cuál sea el gobierno municipal de turno. Si no hay una política de largo aliento, nunca se podrá dejar atrás la mediocridad e improvisación. Los autores de este artículo pudieron tener conocimiento, en 2014, de los proyectos urbanísticos desarrollados por el Plan Metropolitano de Desarrollo Urbano de Lima y Callao al 2035 (PLAM al 2035), en los cuales, por primera vez, el patrimonio arqueológico no solo fue tomado en cuenta, sino que se hizo un estudio detallado de su desarrollo en los diferentes distritos de Lima Metropolitana, lo cual es imprescindible para conceptuar el desarrollo vial y de comunicaciones en un futuro cercano.

\section{Referencias}

Agurto, S. (1984). Lima prehispánica. Lima, Perú: Municipalidad Metropolitana de Lima.

Cárdenas, M. (1971). Huaca Palomino (Valle de Rímac). Fragmentaria Vidriada Fina con Decoración en Colores. Boletín del Seminario de Arqueología. (10), 61-65.

Cárdenas, M. (1973). Cerámica de Transición. Huaca Palomino (Valle del Rímac). Boletín del Seminario de Arqueología, (14), 30-34.

Corbacho, S. (1970). Mate Pirograbado de la Huaca Corpus I. Fundo Pando. Boletín del Seminario de Arqueología, (8), 1-6.

Corbacho, S. (1971). Huaca Corpus I. Fundo Pando. (Informe Preliminar). Boletín del Seminario de Arqueología, (11), 89-93.

Espinoza, P. (2009). Arqueología de Lima: Mateo Salado. Cuadernos del Patrimonio Cultural, (2).

Espinoza, P. (2013). Mateo Salado: El Gran Complejo Arqueológico Tardío de Lima. Arkinka, (215), 96-107.

Figueroa, A. (2007). Resurrección de Mateo Salado. Gaceta Cultural del Perú, junio-julio, (28) 12 13.

Figueroa, A. (2008). Mateo Salado: En Camino a su Revaloración. Gaceta Cultural del Perú. Julio, (34), 32-33.

Guarisco, P. (2012). Informe del Proyecto de Investigación, Conservación y Puesta en Valor de la "Huaca Palomino" y "Huaca Corpus I". Lima, Perú: Ministerio de Cultura.

Hauskeller, M. (2008). ¿Qué es el Arte? Posiciones de la estética desde Platón a Danto. Traducción de José Vicente Borja Caballer. España: Editorial EDITILDE S.L. (Diálogo-Tilde).

Miró Quesada, L. (2014). Residencial Palomino. Arquitecto Luis Miró Quesada Garland. Recuperado de http://arquitectolmqg.blogspot.com/2014/10/29.html

Ministerio de Cultura. (2014). Informe del Proyecto de Investigación, Conservación y Puesta en valor de la "Huaca Corpus I".

Ministerio de Cultura. (2015). Informe del Proyecto de Investigación, Conservación y Puesta en valor de la "Huaca Culebras" y "Huaca Corpus II".

Ramos de Cox, J. (1970). Excavaciones de Unidades Arqueológicas en el Fundo Pando. Boletín del Seminario de Arqueología. (7) 78-82.

Ravines, R. (1985). Inventario de monumentos arqueológicos del Perú. Lima, Perú: Municipalidad de Lima Metropolitana - Instituto Nacional de Cultura.

\section{Agradecimientos}

El programa Lima Milenaria Ciudad de Cultura se realizó gracias a decisión de la Sra. Susana Villarán de la Puente, alcaldesa de Lima Metropolitana, durante los años 2011 y 2014, y al Sr. Pedro Pablo Alayza, Gerente de Cultura. 
devenir Vol. 2, N³3, ENERO - JUNIO 2015, PP. 99-112 - ESTUDIOS I ISSN 2312-7570

UNIVERSIDAD NACIONAL DE INGENIERÍA, LIMA

Los autores quieren agradecer a todo el grupo humano que hizo posible el proyecto de la puesta en valor del patrimonio arqueológico: Piero Guarisco, Gabriela Ugarte, Pilar Riofrío, Sandra Rospigliosi, David de Piérola, Claire Jaureguy, Anita Tavera, Josefina Jiménez, Patricia Mondoñedo, José Díaz, Alex Flores, Rafael Mori, Giannina Cuadros, José Pablo Aliaga, Gianina Comeca, Alexis Rodriguez y Miguel Valqui, Diego Álvarez, Luis Felipe Villacorta y Martín del Carpio. 